\title{
Home-based fecal calprotectin test is expected to play an important role in patients with inflammatory bowel diseases
}

\author{
Young-Ho Kim \\ Department of Gastroenterology, Samsung Medical Center, Sungkyunkwan University School of Medicine, Seoul, Korea
}

\begin{abstract}
Article: Experience of patients with inflammatory bowel disease in using a home fecal calprotectin test as an objective reported outcome for self-monitoring (Intest Res 2018;16:546553)
\end{abstract}

The introduction of anti-tumor necrosis factor agents has changed therapeutic goals from symptomatic remission to mucosal healing in IBD. Mucosal healing can be assessed most accurately with endoscopy. However, endoscopy is invasive and noninvasive biomarkers that can reflect mucosal healing are needed. Fecal calprotectin (FC) is currently the best available surrogate marker for mucosal inflammatory activity in IBD. ${ }^{1,2}$ Sensitivity and specificity for active mucosal inflammation is likely to be higher for FC compared with CRP. ${ }^{3}$

From a clinician's perspective, it is more important to predict inflammation in the small bowel because colonic inflammation can be assessed relatively easily with colonoscopy. Several studies showed that FC reflects small bowel inflammation relatively well, but is better for assessment of colonic inflammation. ${ }^{4,5}$

The FC test also has some drawbacks. There can be a large variation in FC values in stool samples from different bowel movements during the same day or from 2 or 3 consecutive days. The largest differences occurred with increasing concentrations of FC. ${ }^{6-8}$

Received October 3, 2018. Revised October 3, 2018. Accepted October 3, 2018. Correspondence to Young-Ho Kim, Department of Gastroenterology, Samsung Medical Center, Sungkyunkwan University School of Medicine, 81 Irwon-ro, Gangnam-gu, Seoul 06351, Korea. Tel: +82-2-3410-3409,

Fax:+82-2-3410-3849, E-mail: bowelkim@gmail.com
However, the main hurdle for using FC as a biomarker to monitor disease activity is patient reluctance to collect and bring samples to the hospital. In this regard, the research of Wei et al. ${ }^{9}$ is very important and timely. They prospectively enrolled consecutive patients with IBD in clinical remission and assessed FC by using both laboratory and home-based tests. The correlation between the laboratory and home-based tests was good and most patients found the home-based test to be feasible and easy to use and preferred it over the laboratory test.

Home-based tests can overcome the reluctance to use fecal tests. In addition, home-based FC tests can be conducted when patient symptoms deteriorate between outpatient visits, and physicians can use FC test results between outpatient visits and at an outpatient visit to establish the therapeutic plan. Moreover, results of home-based FC tests can be reported to physicians on the internet. Using the results, physicians can predict a relapse and undertake immediate action. This approach would enable telemedicine.

However, there are several issues to consider in using homebased FC tests in actual practice. As the authors pointed out, the patients enrolled in this study had an active desire to use the test, which caused them to overestimate the feasibility and satisfaction with the home-based test. For example, one study showed a significant difference in correlation coefficients 
when stratifying use of the laboratory FC test and the homebased FC test according to education level. ${ }^{10}$ In actual practice, a subset of patients may have difficulty in understanding and using home-based tests. The question is how to sufficiently simplify a home-based FC test to reduce the number of patients who cannot use the test. Another issue is the price. According to this study, $80 \%$ of the participants indicated a strong $(>70 \%)$ probability of using the test if the price was approximately US dollars 20 . The appropriate price can vary depending on the health care system and economic status in each country. The price of home-based FC tests should be affordable even when multiple tests are required.

Home-based FC testing is expected to play an important role for patients with IBD in the future although there are still problems that must be solved. A prospective study to evaluate the effect of home-based FC testing on patient outcomes is required.

\section{FINANCIAL SUPPORT}

The authors received no financial support for the research, authorship, and/or publication of this article.

\section{CONFLICT OF INTEREST}

No potential conflict of interest relevant to this article was reported.

\section{AUTHOR CONTRIBUTION}

Writing and approval of final manuscript: YH Kim.

\section{REFERENCES}

1. Lee KM. Fecal biomarkers in inflammatory bowel disease. Intest Res 2013;11:73-78.

2. Wei SC. Could fecal calprotectin enter mainstream use for diagnosing and monitoring inflammatory bowel disease? Intest Res 2016;14:293-294.

3. Lewis JD. The utility of biomarkers in the diagnosis and therapy of inflammatory bowel disease. Gastroenterology 2011;140:18171826.e2.

4. Arai T, Takeuchi K, Miyamura M, et al. Level of fecal calprotectin correlates with severity of small bowel Crohn's disease, measured by balloon-assisted enteroscopy and computed tomography enterography. Clin Gastroenterol Hepatol 2017;15:56-62.

5. Kawashima K, Ishihara S, Yuki T, et al. Fecal calprotectin more accurately predicts endoscopic remission of Crohn's disease than serological biomarkers evaluated using balloon-assisted enteroscopy. Inflamm Bowel Dis 2017;23:2027-2034.

6. Lasson A, Stotzer PO, Öhman L, Isaksson S, Sapnara M, Strid H. The intra-individual variability of faecal calprotectin: a prospective study in patients with active ulcerative colitis. J Crohns Colitis $2015 ; 9: 26-32$.

7. Moum B, Jahnsen J, Bernklev T. Fecal calprotectin variability in Crohn's disease. Inflamm Bowel Dis 2010;16:1091-1092.

8. Naismith GD, Smith LA, Barry SJ, et al. A prospective single-centre evaluation of the intra-individual variability of faecal calprotectin in quiescent Crohn's disease. Aliment Pharmacol Ther 2013;37:613-621.

9. Wei SC, Tung CC, Weng MT, Wong JM. Experience of patients with inflammatory bowel disease in using a home fecal calprotectin test as an objective reported outcome for self-monitoring. Intest Res 2018;16:546-553.

10. Vinding KK, Elsberg H, Thorkilgaard T, et al. Fecal calprotectin measured by patients at home using smartphones: a new clinical tool in monitoring patients with inflammatory bowel disease. Inflamm Bowel Dis 2016;22:336-344. 